\title{
JANELA PERICÁRDICA TRANSDIAFRAGMÁTICA NO DIAGNÓSTICO DE LESÃO CARDÍACA
}

\author{
TRANSDIAPHRAGMATIC PERICARDICAL WINDOW IN THE DIAGNOSIS OF CARDIAC \\ INJURY
}

\author{
Mario Mantovani,TCBC-SP' ${ }^{1}$ Juliana Pinho Espinola² ; Gustavo Pereira Fraga, TCBC-SP $^{3}$
}

\begin{abstract}
RESUMO: Objetivos: Avaliar a eficácia e as complicações da utilização da janela pericárdica (JP) transdiafragmática no diagnóstico de lesão cardíaca por ferimento penetrante. Método: No período de janeiro de 1994 a dezembro de 2004 a JP foi indicada em 245 casos com suspeita de trauma cardíaco por ferimento penetrante. Em 38 deles (15,5\%) a JP foi realizada através de acesso transdiafragmático, constituindo a população do presente estudo. Resultados: O mecanismo de lesão foi ferimento por projétil de arma de fogo (FPAF) em 26 casos $(68,4 \%)$ e por arma branca (FAB) em 12 casos $(31,6 \%)$. Na maioria dos pacientes $(27$ casos - 71\%) os ferimentos eram múltiplos. A média de RTS foi de 7,32 e a média de PATI foi de 9,8. A JP foi considerada positiva em seis casos $(15,8 \%)$ dos quais cinco $(83 \%)$ por FPAF e um $(17 \%)$ por FAB. Houve um paciente, vítima de múltiplos FPAF, com JP positiva e toracotomia em que não foi identificada lesão cardíaca. A sensibilidade do método foi de $97,4 \%$ e a especificidade de $100 \%$. Em 12 $(31,6 \%)$ pacientes houve associação de lesão com perfuração de víscera oca. Houve um único caso $(2,6 \%)$ de complicação diretamente relacionada ao procedimento, em uma vítima de múltiplos FPAF com lesão perfurada de fígado, estômago e cólon, que evoluiu com pericardite, necessitando posteriormente de drenagem pericárdica, com boa resolução. A mortalidade foi de 7,9\% (três casos), um dos quais em pós-operatório de reparo de lesão cardíaca por coagulopatia/acidose. Conclusão: A JP trandiafragmática é um procedimento que permite o rápido diagnóstico de lesão cardíaca em pacientes nos quais a prioridade é a laparotomia. Trata-se de método de fácil realização e alta sensibilidade (Rev. Col. Bras. Cir. 2006; 33(1): 29-34).
\end{abstract}

Descritores: Coração; Técnicas de janela pericárdica/ efeitos adversos; Ferimento penetrantes/ diagnóstico; Traumatismos cardíacos.

\section{INTRODUÇÃO}

A lesão cardíaca por ferimento penetrante é uma causa freqüente de óbito em trauma e deve ser rapidamente diagnosticada ${ }^{1,2}$. As poucas vítimas de ferimentos penetrantes na área de Sauer-Murdock (ATLS $\left.{ }^{\circledast}\right)^{3}$, que chegam ao hospital com vida devem ser prontamente investigadas, pois $20 \%$ a $40 \%$ destes pacientes apresentam-se inicialmente com estabilidade hemodinâmica, e podem evoluir rapidamente para a morte por tamponamento cardíaco ou sangramento $0^{4-10}$.

A janela pericárdica (JP) é considerada por muitos autores o padrão ouro para o diagnóstico de ferimento cardíaco, visto que rapidamente identifica ou exclui a presença de lesão, e é realizada com facilidade técnica, alta sensibilidade e especificidade, além de mínima morbidade ${ }^{1,2,5,6,8,11}$. A visualização direta do pericárdio assegura se há sangue ou coágulo no saco pericárdico e, então, a incisão pode ser facilmente estendida em uma toracotomia mediana ou lateral. Porém, se o resultado da JP for negativo, com saída de líquido claro, a incisão cirúrgica pode ser fechada e a necessidade de toracotomia é excluída.

Alguns pacientes que são vítimas de ferimento penetrante apresentam lesões tóraco-abdominais associadas.
Nesses casos, o paciente deve ser submetido a uma laparotomia exploradora para investigação cirúrgica da cavidade abdominal e pode ser realizada a janela pericárdica transdiafragmática (JPTD), descrita inicialmente por Garrison et $a l^{10}$, em 1982, a fim de promover um diagnóstico mais rápido e seguro de lesão cardíaca.

O objetivo do presente estudo é verificar os resultados deste procedimento realizado em pacientes atendidos pela Disciplina de Cirurgia do Trauma do Departamento de Cirurgia da Faculdade de Ciências Médicas da Unicamp, analisando o mecanismo do trauma, a incidência de lesão cardíaca, a relação do número de ferimentos com a presença da lesão cardíaca, a sensibilidade e especificidade do método, e as respectivas taxas de complicações.

\section{MÉTODO}

A Disciplina de Cirurgia do Trauma tem como protocolo a obrigatoriedade de investigação de lesão cardíaca em todo paciente com trauma penetrante na área de SauerMurdock, em que o orifício do ferimento ou a trajetória do projétil ou do objeto contundente (arma branca), estejam localizados na projeção anterior ou posterior do tórax nesta

1. Professor Titular e Chefe da Disciplina de Cirurgia do Trauma da FCM - Unicamp.

2. Aluna de Graduação e da Liga do Trauma da FCM - Unicamp.

3. Professor Assistente Doutor da Disciplina de Cirurgia do Trauma da FCM - Unicamp e do Departamento de Cirurgia do Hospital Municipal "Dr. Mário Gatti”, Campinas - SP.

Recebido em 17/08/2005

Aceito para publicação em 19/10/2005

Conflito de interesses: nenhum

Fonte de financiamento: FAPESP

Trabalho realizado na Disciplina de Cirurgia do Trauma do Departamento de Cirurgia da Faculdade de Ciências Médicas (FCM) da Universidade Estadual de Campinas (Unicamp). 
Figura 1 - Organograma com o número de casos em que foi realizada a JP e o total de lesões cardíacas.

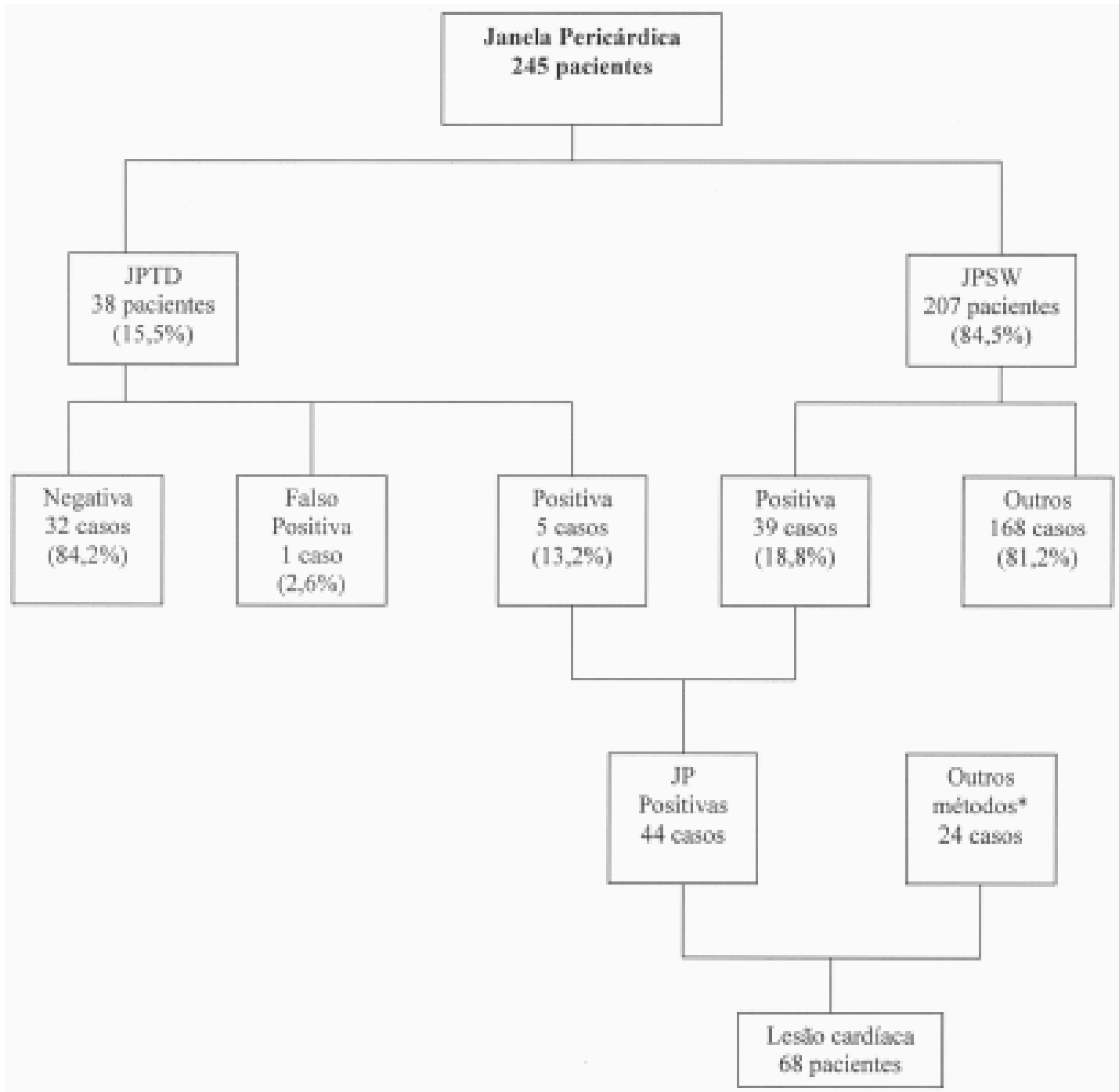

* outros métodos diagnósticos: indicação clínica de toracotomia com identificação de lesão no intra-operatório; ecocardiograma; videotoracoscopia.

área de risco, sendo rotina inclusive em pacientes considerados "assintomáticos".

Os pacientes com instabilidade hemodinâmica são reanimados conforme a seqüência do $\operatorname{ATLSâ}^{3} \mathrm{e}$, caso não apresentem melhora, são conduzidos ao centro cirúrgico. Os pacientes com estabilidade hemodinâmica e ferimento na área de risco são investigados com um dos seguintes métodos: ecocardiografia bidimensional, JP e, mais recentemente, a videotoracoscopia.

A JP é indicada quando não há disponibilidade de realizar a ecocardiografia bidimensional, ou quando o paciente tem alguma outra indicação cirúrgica, como ferimento associado no abdome ou suspeita de lesão diafragmática. A JP é um procedimento realizado sob anestesia geral no centro cirúrgico e, na maioria das vezes, é feita através de acesso subxifóide (JPSX), precedendo a laparotomia. Em algumas situações o cirurgião julga prioritária a realização da laparotomia, baseado no mecanismo e condições clínicas do paciente, deixando a JP para ser realizada na seqüência. Nestas situações, o acesso transdiafragmático (JPTD) torna-se uma excelente opção, conforme a experiência do serviço.

Já com exposição da cavidade abdominal através de laparotomia mediana, inicialmente procede-se ao controle de grandes hemorragias de estruturas abdominais e ao controle provisório de contaminação peritoneal. Ao nível do ictus diafragmático, o diafragma é pinçado com pinças de Allis lateralmente à porção tendinosa e rebatido inferiormente para deslocar o diafragma e o saco pericárdico do ápice do coração. Uma incisão vertical de aproximadamente $2 \mathrm{~cm}$ é feita na linha média da porção tendinosa do diafragma que permite a abordagem do saco pericárdico e resulta na identificação do líquido livre, sangue ou coágulo pelo orifício. A JPTD é interpretada como negativa na ausência de sangue ou coágulo, e a incisão da pericardiotomia é fechada. Quando não há saída de sangue pelo orifício da pericardiotomia, coloca-se uma sonda de nelaton dentro do saco pericárdico e injeta-se $200 \mathrm{ml} \mathrm{de}$ solução salina morna e observa-se o resultado: é considerado negativo quando o líquido retorna sem característica de 
sangramento. Se não houver retorno do líquido, ou vier com sangramento associado, o resultado é considerado positivo para lesão de coração e/ou grandes vasos. Esta lavagem intrapericárdica diagnóstica tem sido por nós realizada nos casos em que não ocorreram lesões, mostrando-se efetiva. Uma JPTD positiva requer imediata esternotomia ou toracotomia ântero-lateral a fim de reparar a lesão cardíaca.

No período de janeiro de 1994 a dezembro de 2004 foram operados 2147 casos de trauma no nosso serviço. A JP foi indicada em 245 pacientes (11,4\%), e em 38 deles $(15,5 \%)$, foi utilizada a técnica transdiafragmática (JPTD) (Figura 1). Neste mesmo período, ocorreram 68 casos de lesão cardíaca, sendo todos registrados em banco de dados com programa Epi-Info 6.04, com as seguintes informações: identificação, sexo, idade, data de admissão, mecanismo de trauma e número de lesões, dados fisiológicos, índices de trauma, lesões anatômicas, sensibilidade e especificidade da JPTD, incidência de lesão cardíaca, tratamento e evolução dos pacientes.

Em todos os pacientes foram utilizados os seguintes índices de trauma: Escore de Trauma Revisado (Revised Trauma Score ou RTS), Índice de Gravidade da Lesão (Injury Severity Score ou ISS), Classificação das Lesões Cardíacas (OIS), TRISS (Probabilidade de Sobrevida) e Índice de Trauma Abdominal (Abdominal Trauma Index ou ATI) ${ }^{12-16}$. O RTS é um índice fisiológico que utiliza para cálculo a escala de coma de Glasgow, a pressão arterial sistólica e a frequiência respiratória $^{12}$. O ISS é um índice anatômico que avalia cada segmento corpóreo: considera-se a lesão mais grave, seleciona-se os três segmentos que apresentaram lesão com maior pontuação e eleva-se cada um destes valores ao quadrado; a soma dos mesmos resulta no ISS ${ }^{13}$. O ISS varia de 0 a 75 , com pontuação de gravidade crescente. As lesões cardíacas foram agrupadas de acordo com a classificação da Associação Americana de Cirurgia do Trauma ${ }^{14}$. O TRISS é um índice misto que é obtido após associar o RTS, o ISS, a idade da paciente (menor ou igual a 54 anos ou maior que 54 anos) e o tipo de traumatismo (fechado ou penetrante), sendo que o valor obtido representa a probabilidade de sobrevida estimada para o traumatizado ${ }^{15}$. O ATI é outro índice anatômico onde o escore de lesão de cada órgão intra-abdominal é o produto do fator de risco pela gravidade da lesão neste órgão, e a soma dos escores de todos os órgãos lesados representa o $\mathrm{ATI}^{16}$.

\section{RESULTADOS}

Dentre os 38 pacientes que foram submetidos à JPTD, trinta e três $(86,8 \%)$ eram do sexo masculino e cinco $(13,2 \%)$ do sexo feminino, com idade variando de 15 a 58 anos (média de 28,8 anos). O ferimento foi causado por projétil de arma de fogo (FPAF) em 26 pacientes $(68,4 \%)$, enquanto 12 pacientes $(31,6 \%)$ foram vítimas de ferimento por arma branca (FAB). Onze pacientes $(29 \%)$ tiveram ferimento único na parede torácica e/ou abdominal e 27 (71\%) foram vítimas de múltiplos ferimentos.

Em relação às condições fisiológicas na admissão hospitalar, 30 pacientes $(78,9 \%)$ apresentaram pressão arterial sistólica (PAS) maior ou igual a $90 \mathrm{mmHg}$ e outros oito $(21,1 \%)$ foram considerados instáveis hemodinâmicamente, com PAS
Tabela 1 - Número de casos conforme órgão e estrutura abdominal lesada.

\begin{tabular}{lcc}
\hline Órgão/estrutura lesada & $\mathbf{N}^{\mathbf{0}}$ de casos & $\mathbf{( \% )}$ \\
\hline diafragma & 16 & 42,1 \\
fígado & 14 & 36,8 \\
estômago & 10 & 26,3 \\
jejuno/íleo & 7 & 18,4 \\
cólon & 7 & 18,4 \\
baço & 3 & 7,9 \\
grandes vasos abdominais & 3 & 7,9 \\
pâncreas & 1 & 2,6 \\
rim & 1 & 2,6 \\
ureter & 1 & 2,6 \\
\hline
\end{tabular}

$<90 \mathrm{mmHg}$, com resposta satisfatória à reposição volêmica. $\mathrm{O}$ RTS variou de 1,46 a 7,84, com média de 7,32. Vinte e cinco $(65,8 \%)$ pacientes apresentaram RTS máximo de 7,84 na admissão.

Em nenhum paciente foi realizada punção de Marfan (pericardiocentese) antes da JP. Os órgãos e estruturas com lesões identificadas e reparadas na laparotomia exploradora estão listados na Tabela 1. Em 12 pacientes $(31,6 \%)$ havia perfuração de víscera oca. Em 18 casos $(47,4 \%)$ a laparotomia foi considerada como não-terapêutica. O ATI variou de 0 a 63, com média de 9,83 . Trinta e quatro pacientes $(89,5 \%)$ apresentaram hemo ou pneumotórax e foram submetidos à drenagem torácica. O ISS variou de dois a 50, com média de 18.

O resultado da JPTD foi considerado negativo em 32 casos $(84,2 \%)$. Em seis pacientes $(15,8 \%)$ a JPTD foi interpretada como positiva, e imediatamente indicada a toracotomia. A Tabela 2 mostra os achados e evolução dos pacientes com JPTD positiva.

Um dos pacientes (2,6\%), obeso, apresentava múltiplos FPAF, com hemotórax e JPTD interpretada como positiva, mas durante a toracotomia não foi identificada lesão cardíaca, o que foi considerado um resultado falso positivo.

A utilização do método evidenciou sensibilidade de $100 \%$, com especificidade de $96,9 \%$, acurácia de $97,4 \%$, valor preditivo positivo de $83,3 \%$ e valor preditivo negativo de $100 \%$.

A morbidade na população estudada foi de $39,5 \%$ (15 casos). Foram mais freqüentes as complicações pulmonares (sete casos). Complicação diretamente relacionada à JPTD foi diagnosticada em um paciente $(2,6 \%)$, vítima de múltiplos FPAF, com hemotórax, lesão de diafragma, fígado, estômago e cólon, com grande contaminação da cavidade peritoneal, com JPTD negativa. Apesar dos cuidados quanto à contaminação no intra-operatório, este paciente evoluiu com pericardite, necessitou ser re-operado para drenagem do saco pericárdico no $28^{\circ}$ dia pós-operatório, e permaneceu internado por mais cinco dias.

A mortalidade foi de 7,9\% (três casos). Nenhum dos óbitos teve relação com o procedimento JPTD, e um dos pacientes morreu no $1^{\circ}$ dia pós-operatório de reparo de lesão cardíaca, devido a hemorragia e coagulopatia. 
Tabela 2 - Achados e evolução dos seis pacientes com JPTD positiva.

\begin{tabular}{lllccl}
\hline Mecanismo & Quantidade & \multicolumn{1}{c}{ Incisão } & Local & Grau & Evolução \\
\hline FPAF & múltiplos & Toracotomia ALE & VE & 2 & Alta (14 dias) \\
FPAF & múltiplos & Toracotomia ALE & Pericárdio & 1 & Alta (10 dias) \\
FAB & único & Toracotomia ALE & VD & 2 & Alta $(5$ dias) \\
FPAF & múltiplos & Esternotomia & VE & 5 & Óbito (1 dia) \\
FPAF & múltiplos & Toracotomia ALE & VD & 3 & Alta (31 dias) \\
FPAF & múltiplos & Toracotomia ALE & - & Falso positivo & Alta (8 dias) \\
\hline
\end{tabular}

FPAF: ferimento por projétil de arma de fogo; FAB: ferimento por arma branca; ALE: ântero-lateral esquerda; VE: ventrículo esquerdo; VD: ventrículo direito.

A mortalidade do trauma cardíaco penetrante no serviço nos 68 pacientes tratados nos últimos onze anos foi de $16,2 \%$. No presente estudo, o TRISS teve uma média de 0,94 .

\section{DISCUSSÃO}

A grande maioria dos pacientes com trauma cardíaco penetrante morreu antes do atendimento hospitalar. A Disciplina de Cirurgia do Trauma tratou nos últimos 11 anos, 68 pacientes com lesão cardíaca, o que resulta numa média de 6,2 casos por ano. Na região de Campinas, o HC-Unicamp é um dos poucos centros de referência para tratamento destes casos, e somados aos casos do Hospital Municipal "Dr. Mário Gatti" e Hospital e Maternidade "Celso Pierro", da PUC-Campinas, estima-se uma média de 12 casos de lesão cardíaca atendidos por ano. Em estudo realizado no Instituto Médico Legal de Campinas nos anos de 2000 e 2001 foram registradas 1976 necrópsias por causas externas, e destas, 1294 casos $(65,5 \%)$ por trauma penetrante, sendo a lesão cardíaca identificada em 296 necrópsias $(22,9 \%)$ e apenas 18 pacientes $(6 \%)$ foram submetidos à toracotomia, comprovando a elevada mortalidade dos traumas cardíacos penetrantes ${ }^{17}$.

Aproximadamente $20 \%$ dos pacientes que são admitidos no hospital com trauma cardíaco não apresentam sintomas ou sinais clínicos sugestivos de lesão6,9. É importante que as equipes de urgência e serviços de trauma tenham protocolos para investigação sistemática destes pacientes assintomáticos ${ }^{2}$. Em particular, nosso serviço utiliza o que o $\mathrm{ATLS}^{\circledR}$ recomenda, ou seja, que todo paciente com ferimento penetrante na área de Sauer-Murdock seja investigado, enquanto outros serviços adotam a área de Ziedler como área de risco $^{1-3,9}$.

A ecocardiografia bidimensional é um método de investigação, com elevada sensibilidade e especificidade, além da vantagem de não ser invasivo ${ }^{18-21}$. Porém, muitos destes ferimentos também estão localizados na região tóraco-abdominal, com indicação cirúrgica para investigação de lesão diafragmática ou outras lesões associadas. Nestas situações, a videotoracoscopia é um método diagnóstico invasivo que pode ser realizado, inclusive com possibilidade de avaliação de lesão cardíaca e até mesmo realização de janela pericárdica através da vídeocirurgia ${ }^{22,23}$. Entretanto, muitos pacientes têm lesões abdominais associadas, o que torna evidente a indicação de laparotomia. Na presente casuística $71 \%$ dos casos apresentavam múltiplos ferimentos.

A literatura atribui a Arom et $l^{24}$ a utilização rotineira de janela pericárdica subxifóidea na investigação de lesões cardíacas. Estes autores descreveram este procedimento realizado inclusive sob anestesia local. A maioria das casuísticas sobre janela pericárdica mostra a preferência da realização da técnica subxifóidea à realizada por via transdiafragmática, principalmente com o objetivo de evitar contaminação do pericárdio por eventual lesão do trato digestivo associada ${ }^{5,6,9,11}$. A Tabela 3 mostra a freqüência com que o acesso transdiafragmático foi utilizado em algumas séries.

Conforme descrito inicialmente por Garrison et $\mathrm{l}^{10}$, a JPTD está indicada naqueles pacientes com ferimentos na transição tóraco-abdominal nos quais há indicação de laparotomia. Em 60 casos avaliados, $53(88,3 \%)$ por trauma penetrante e sete $(11,7 \%)$ por trauma contuso, as indicações para a realização da JPTD foram: proximidade de penetração do agente contundente ao saco pericárdico ( 44 casos $-73,3 \%$ ) ou hipotensão sem causa definida $(16 \text { casos }-26,7 \%)^{10}$.

$\mathrm{Na}$ literatura, o índice de positividade da JP varia muito de um serviço para outro, com maior parte das casuísticas mostrando taxas de 18 a $30 \% \%^{5-9}$. Analisando apenas publicações que citam a positividade de JPTD, Garrison et $_{\text {al }}{ }^{10}$ encontraram uma taxa de $31,7 \%$ e Miller et $\mathrm{al}^{5}$ de $25 \%$. No presente

Tabela 3 - Número de casos e porcentagens da realização da JPTD.

\begin{tabular}{lcc}
\hline Autores & $\mathbf{N}^{\text {o. }}$ de casos de jp & $\mathbf{N}^{\circ}$ de casos e porcentagem de jptd \\
\hline Miller et al, $1987^{5}$ & 104 & $16(15,4 \%)$ \\
Brewster et al, $1988^{6}$ & 108 & $39(36,1 \%)$ \\
Mayor-Davies et al, $1990^{11}$ & 10 & 0 \\
Prado et al, 1995 & 41 & $2(4,9 \%)$ \\
Grewal et al, 1995 & 122 & 0 \\
Presente casuística & 245 & $38(15,5 \%)$ \\
\hline
\end{tabular}


estudo, a positividade da JPTD foi de $15,8 \%$, com elevada sensibilidade $(97,4 \%)$ e especificidade $(100 \%)$.

No serviço, a JP sempre é realizada sob anestesia geral, no centro cirúrgico. Embora alguns autores realizem o procedimento sob anestesia local, compartilhamos da opinião de Brewster et $a l^{6}$, que também não aceitam que a JP seja realizada na sala de urgências nem sob anestesia local, e descrevem um tempo médio de 36 minutos de duração do ato operatório no centro cirúrgico quando o resultado era negativo. Obviamente que a JPTD exige anestesia geral, pois é realizada no transcorrer de uma laparotomia.

Como a JP é um método com elevada sensibilidade, qualquer descuido técnico durante a realização do procedimento pode ocasionar um resultado falso-positivo, com a conseqüente realização de uma toracotomia não terapêutica. Isto ocorreu com um paciente $(2,6 \%)$ na presente casuística. Miller et $a l^{5}$ também descreveram um caso $(1,7 \%)$ em que havia sangue no saco pericárdico e não foi detectada lesão cardíaca durante a cirurgia, porém não fizeram referência se o acesso foi TD. Garrison et $\mathrm{l}^{10}$, utilizando a JPTD, observaram que 17 dos 19 pacientes $(89,5 \%)$ com resultado positivo tinham lesão miocárdica. Outros dois pacientes $(10,5 \%)$ tinham lesão do pericárdio sem acometimento do miocárdio, o que é interpretado como lesão grau I, e isto só foi identificado na toracotomia. Grewal et $a l^{7}$ realizaram JPSX em 122 casos, com 26 exames positivos $(21,3 \%)$. Destes, em dois pacientes $(7,7 \%)$ havia lesão cardíaca grau I. O tratamento conservador de ferimentos cardíacos em pacientes estáveis, embora descrito desde 1943 por Blalock e Ravitch ${ }^{25}$, é uma conduta de exceção, sendo que a maioria dos artigos da literatura ainda preconiza a cirurgia precoce para reparo das lesões cardíacas ${ }^{5,6,8,9}$. Recentemente, Navsaria e $\mathrm{Nicol}^{26}$, em estudo prospectivo, observaram que $\operatorname{dez}(71,4 \%)$ de 14 pacientes estáveis com JP positiva tinham lesões cardíacas menores (graus I a III) identificadas durante a esternotomia, sendo que este procedimento mais invasivo poderia ter sido evitado. Assim sendo, em outro grupo de sete pacientes, a maioria $(85,7 \%)$ vítimas de FAB, e tratados em média 48 horas após o trauma, foi realizado tratamento com JP, drenagem do saco pericárdico e observação. Estes pacientes evoluíram bem, com média de internação de 4,7 dias $^{26}$. No pre- sente estudo, um dos pacientes apresentou lesão cardíaca grau I, o que justificou a toracotomia, que poderia ser interpretada como não terapêutica, embora tenha evitado eventual evolução para tamponamento cardíaco.

Lesões abdominais associadas foram identificadas em 20 casos (52,6\%). No serviço, a investigação cirúrgica de lesão diafragmática é imperativa em todo trauma penetrante na região tóraco-abdominal e, na indisponibilidade de realizar videotoracoscopia ou videolaparoscopia, como o ocorrido na casuística, indica-se a laparotomia exploradora, o que acaba resultando em elevadas taxas (18 casos $-47,4 \%$ ) de procedimento não terapêutico. Deve-se ter cuidado especial quando há perfuração de víscera oca (presente em 12 casos - 31,6\%), principalmente a fim de evitar contaminação na região subfrênica em que é feito o acesso TD. Isto não contra-indica a JPTD e outros autores não encontraram complicações decorrentes de lesão gastrointestinal associada à $\mathrm{JP}^{5,6,10}$. Entretanto, no presente estudo, um paciente com lesão de estômago e cólon, apesar de todas as precauções tomadas no intraoperatório, evoluiu com pericardite, com tempo de internação prolongado e necessidade de re-operação para drenagem pericárdica, com boa resolução após essa intervenção. A conduta do nosso serviço é não realizar a JPTD quando houver grande contaminação da cavidade peritoneal por conteúdo fecal ou entérico e, no caso citado, incorretamente, o cirurgião optou pelo método. Nestes casos, não realizamos a JPTD e sim a JP clássica, com os cuidados relativos à contaminação local.

Na presente casuística, os três casos de óbito $(7,9 \%)$ não tiveram associação com a realização da JPTD.

Conclui-se que a JPTD é um procedimento que permite o rápido diagnóstico de lesão cardíaca em pacientes nos quais a prioridade é a laparotomia. A técnica cirúrgica deve ser cuidadosa especialmente em ferimentos múltiplos a fim de evitar resultados falso-positivos e quando há contaminação da cavidade peritoneal, a fim de evitar complicações infecciosas no pericárdio (2,6\% na presente casuística). Trata-se de método de fácil realização e alta sensibilidade, devendo fazer parte dos métodos de investigação diagnóstica de domínio do cirurgião do trauma.

\begin{abstract}
Background: This retrospective study was undertaken to evaluate the efficacy and the complications of the transdiaphragmatic pericardial window (TPW) on the diagnosis of heart injury caused by penetrating wounds. Methods: During the period of january, 1994 to december, 2004, the pericardial window $(P W)$ was indicated in 245 suspicious cases of cardiac trauma caused by penetrating wounds. In 38 of them (15.5\%), PW was accomplished through trandiaphragmatic approach, composing the population of this study. Results: The trauma mechanism was gunshot in 26 patients (68.4\%) and stab wounds in $12(31.6 \%)$. Most of the patients presented multiple wounds (27 cases - 71\%). The RTS average was 7.32 and the PATI average was 9.8. The $P W$ was considered positive in 6 cases $(15.8 \%)$ of those: $5(83 \%)$ by gunshot e $1(17 \%)$ by stab wounds. There was one patient, with multiple gunshot wounds, that present a positive PW but thoracotomy showed no cardiac injury. The sensibility of TPW was $97.4 \%$ and the specificity was $100 \%$. In $12(31.6 \%)$ patients there was association between injury and a perforated hollow gut. There was a single case (2.6\%) of complication directly associated to the procedure, being this patient a victim of multiple gunshot wounds with perfurated liver, stomach and colon, who developed pericarditis and needed pericardial drainage, later with good outcome. The mortality rate was $7.9 \%$ (3 cases), and one of this patients died in because of postoperative acidosis and coagulopathy. Conclusion: The TPW procedure allows rapid diagnostic of cardiac injury in patients who have the lapatotomy as a priority. It is an easy method with high sensibility.
\end{abstract}

Key words: Heart; Pericardial window techniques/adverse effects; Wounds penetrating/diagnosis; Heart injuries. 


\section{REFERÊNCIAS}

1. Razuk Filho A. Ferimentos cardíacos penetrantes: análise dos fatores relacionados à mortalidade [dissertação]. São Paulo (SP): Faculdade de Ciências Médicas da Santa Casa de São Paulo; 1999.

2. Baccarin V, Rizoli SB, Vieira RW, et al. Ferimentos cardíacos penetrantes: experiência com 21 casos. Rev Col Bras Cir. 1992;19(3):112-5.

3. American College of Surgeons Committee on Trauma. Advanced Trauma Life Suppoer Course Manual. American College of Surgeons, $3^{\text {rd }}$ ed; 1997.

4. Nunes R, Higa C, Saito E, et al. Trauma cardíaco. In: Freire E, editor. Trauma: a doença dos séculos. Rio de Janeiro: Atheneu; 2001. p. 1395-430.

5. Miller FB, Bond SJ, Shumate CR, et al. Diagnostic pericardial window. A safe alternative to exploratory thoracotomy for suspected heart injuries. Arch Surg. 1987;122(5):605-9.

6. Brewster SA, Thirlby RC, Snyder WH $3^{\text {rd }}$. Subxiphoid pericardial window and penetrating cardiac trauma. Arch Surg. 1988;123(8):937-41.

7. Grewal H, Ivatury RR, Divakar M, et al. Evaluation of subxiphoid pericardial window used in the detection of occult cardiac injury. Injury. 1995;26(5):305-10.

8. Duncan AO, Scalea TM, Sclafani SJA, et al. Evaluation of occult cardiac injuries using subxiphoid pericardial window. J Trauma. 1989;29(7):955-9; discussion 959-60.

9. Prado PA, Saad Júnior R, Rasslan S: Pericardical Window in the diagnosis of cardiac injuries. Rev Col Bras Cir. 1995;22(6):30712.

10. Garrison RN, Richardson JD, Fry DE. Diagnostic transdiaphragmatic pericardiotomy in thoracoabdominal trauma. J Trauma. 1982;22(2):147-9.

11. Mayor-Davies JA, Britz RS. Subxiphoid pericardial windows helpful in selected cases. J Trauma. 1990;30(11):1399-401.

12. Champion HR, Sacco WJ, Copes WS, et al. A revision of the trauma score. J Trauma. 1989;29(5):623-9.

13. Baker SP, O'Neill B, Haddon W Jr, et al. The injury severity score: a method for describing patients with multiple injuries and evaluating emergency care. J Trauma. 1974;14(3):187-96.

14. Moore EE, Malangoni MA, Cogbill TH, et al. Organ injury scaling IV: thoracic vascular, lung, cardiac, and diaphragm. J Trauma. 1994;36(3):299-300.

15. Boyd CR, Tolson MA, Copes WS. Evaluating trauma care: the TRISS method. J Trauma. 1987;27(4):370-8.
16. Borlase BC, Moore EE, Moore FA. The abdominal trauma index - a critical reassessment and validation. J Trauma. 1990; 30(11):1340-4

17. Fraga GP, Heinzl LR, Longhi BS, et al. Trauma cardíaco: estudo de necropsias. Rev Col Bras Cir. 2004; 31(6):386-90.

18. Asensio JA, Soto SN, Forno W, et al. Penetrating cardiac injuries: a complex challenge. Injury. 2001;32(7):533-43.

19. Jimenez E, Martin M, Krukenkamp I, et al. Subxiphoid pericardiotomy versus echocardiography: a prospective evaluation of the diagnosis os occult penetrating cardiac injury. Surgery. 1990;108(4):676-9; discussion 679-80.

20. Meyer DM, Jessen ME, Grayburn PA. Use of echocardiography to detect occult cardiac injury after penetrating thoracic trauma: a prospective study. J Trauma. 1995;39(5):902-7; discussion 907-9.

21. Rozycki GS, Feliciano DV, Ochsner MG, et al. The role of ultrasound in patients with possible penetrating cardiac wounds: a prospective multicenter study. J Trauma. 1999;46(4):543-51; discussion 551-2.

22. Morales CH, Salinas CM, Henao CA, et al. Thoracoscopic pericardial window and penetrating cardiac trauma. J Trauma. 1997;42(2):273-5.

23. Pons F, Lang-Lazdunski L, de Kerangal X, et al.: The role of videothoracoscopy im management of precordial thoracic penetrating injuries. Eur J Cardiothorac Surg. 2002;22(1):712.

24. Arom KV, Richardson JD, Webb G, et al. Subxiphoid pericardial window in patients with suspected traumatic pericardial tamponade. Ann Thorac Surg. 1977;23(6):545-9.

25. Blalock A, Ravitch M. A consideration of the nonoperative treatment of cardiac tamponade resulting from wounds of the heart. Surgery. 1943;14(2):157-62.

26. Navsaria PH, Nicol AJ. Haemopericardium in stable patients after penetrating injury: is subxiphoid pericardial window and drainage enough? A prospective study. Injury. 2005;36(6):74550 .

Endereço para correspondência:

Dr. Gustavo Pereira Fraga

Avenida Coronel Silva Telles, 211 ap. 3

Cambuí - CAMPINAS - SP

CEP: 13024-000

e-mail:fragagp@uol.com.br 